\title{
Gender-specific Prevalence and Influencing Factors of Ideal Cardiovascular Health in Chinese Rural Population: The Henan Rural Cohort Study
}

\author{
Xueyan Wu \\ Zhengzhou University \\ Xiaotian Liu \\ Zhengzhou University \\ Zhicheng Luo \\ Zhengzhou University \\ Miaomiao Niu \\ Zhengzhou University \\ Yaling He \\ Zhengzhou University \\ Jian Hou \\ Zhengzhou University \\ Zhenxing Mao \\ Zhengzhou University \\ Wenqian Huo \\ Zhengzhou University \\ Linlin Li \\ Zhengzhou University \\ Chongjian Wang ( $\nabla$ tjwcj2005@126.com ) \\ Zhengzhou University https://orcid.org/0000-0001-5091-6621
}

\section{Research article}

Keywords: Ideal cardiovascular health, Health behaviors, Health factors, Prevalence, Influencing factors, Rural population

Posted Date: July 7th, 2020

DOI: https://doi.org/10.21203/rs.3.rs-32403/v1

License: (c) (i) This work is licensed under a Creative Commons Attribution 4.0 International License. Read Full License 


\section{Abstract}

Background: The American Heart Association define a new concept of ideal cardiovascular health (ICH), which was widely used. However, the prevalence of $\mathrm{ICH}$ according to the definition of Chinese rural population is unclear. The study aimed to estimate prevalence and influencing factors of ICH in rural areas of China.

Methods: This study included 35081 participants (13711 men and 21370 women)aged 18 to 79 years from "the Henan Rural Cohort study". The cardiovascular health (CVH) metrics, the ICH scores, the ideal health behaviors (IHB) scores and ideal health factors (IHF)scores were evaluated in Chinese rural adults. Each $\mathrm{CVH}$ metrics divided into ideal and non-ideal including diet, physical activity, smoking, BMI, blood pressure, total cholesterol and fasting plasma glucose. The influencing factors of ideal CVH (ICH scores $\geq 5$ ), ideal HB (IHB scores $>3$ ) and ideal HF (IHF scores $>3$ ) were explored by logistic regression analysis.

Results: The age-standardized mean scores of the ICH was 4.30(3.62 in men and4.69 in women). Prevalence of ICH metrics about ideal physical activity was the highest (91.37\%), while about ideal diet was lowest (0.48\%).The agestandardized prevalence of population who with 7 scores of $\mathrm{ICH}$ was $0.10 \%(0.07 \%$ in men and $0.11 \%$ in women). The agestandardized prevalence of Ideal CVH (ICH scores $\geq 5$ ) was $48.86 \%$ (28.26\% in men and $60.70 \%$ in women). The agestandardized prevalence of 4 of IHB scores and 4 of IHF scores were $0.16 \%(0.17 \%$ in men and $0.16 \%$ in women) and $33.26 \%$ (10.06\% in men and $46.60 \%$ in women), respectively. Further, apart from IHB scores, age had an inverse relationship with the ICH scores and IHF scores in total populations and women. Much older, men, low family income, current drinking and abdominal obesity were independently associated with ICH in Chinese rural adults.

Conclusions: The percentage of ideal cardiovascular health is extremely low in rural China. There is an increasing need for primordial prevention of unhealthy lifestyles and monitoring high risk factors to promote cardiovascular health in rural China.

\section{Clinical Trial Registration}

The Henan Rural Cohort Study has been registered at Chinese Clinical Trial Register (Registration number: ChiCTR-OOC15006699). Date of registration: 2015-07-06.http://www.chictr.org.cn/showproj.aspx?proj=11375

\section{What Is Already Known On This Topic?}

Previous studies have explored the prevalence and influencing factors of ideal cardiovascular health, but evidence on the ideal cardiovascular health remains uncertain and limited in China, especially in rural population.

\section{What Does This Study Add?}

The results of this study showed that the prevalence of ideal cardiovascular health $(\mathrm{ICH})$ is extremely low, and the status of health behaviors was worse than health factors in Chinese rural adults. Further, the ideal physical activity (91.37\%) was the highest prevalence of $\mathrm{ICH}$ metrics, while the ideal diet $(0.48 \%)$ was the lowest prevalence of $\mathrm{ICH}$ metrics. In addition, apart from ideal health behaviors (IHB) scores, age had an inverse relationship with the ICH scores and ideal health factors (IHF) scores in total populations and women. Much older, men, low family income, current drinking and abdominal obesity were independent influencing factors for $\mathrm{ICH}$ in Chinese rural adults.

\section{Background}

Cardiovascular disease (CVD) is now the leading cause of premature mortality and disability in world in noncommunicable diseases ${ }^{1,2}$. The national disability-adjusted life-years (DALYs) among non-communicable disease 
causes were estimated and more than 17 million people died prematurely by CVD in $2017^{1}$. With rapid economic development, urbanization, and an aging population, CVD has become the leading cause of death in China. Compared with western countries, China has higher age-adjusted mortality from CVD ${ }^{3}$. Aging and population growth will increase cardiovascular disease by more than a half over the coming 20 years and there would be an increase of approximately 7.7 million cardiovascular deaths in China from 2010 to $2030^{4}$. Several studies have suggested the risk factors of CVD, including elevated blood pressure, high total cholesterol, diabetes, obesity/overweight and smoking ${ }^{3,5-7}$.

In 2010, ideal cardiovascular health (ICH) has been formulated by the American Heart Association (AHA) to reduce deaths from all CVDs, and to improve the cardiovascular health of the population as a whole, which is defined as the simultaneous presence of four ideal health behaviors (nonsmoking status, ideal body mass index (BMI), regular physical activity (PA), and healthy diet) and four ideal health factors (ideal smoking status, untreated total cholesterol (TC) $<5.18$ $\mathrm{mmol} / \mathrm{L}$, untreated blood pressure (BP) $<120 /<80 \mathrm{~mm} \mathrm{Hg}$, and untreated fasting plasma glucose (FPG) $<5.6 \mathrm{mmol} / \mathrm{L}$ ) in the absence of cardiovascular disease history. Smoking appears on both lists of health behaviors and health factors due to its importance for health promotion ${ }^{8}$.

Previous studies have indicated the inverse association between the number of ICH metrics and the risks of hypertension ${ }^{9,10}$, type 2 diabetes mellitus ${ }^{11,12}$, CVDevents (stroke, heart failure, myocardial infarction, and fatal coronary disease) ${ }^{13-20}$, CVD mortality ${ }^{21,22}$, all-cause mortality ${ }^{10,23}$ and concer ${ }^{24,25}$. However, most of the previous studies were conducted in western countries and Chinese urban areas ${ }^{26-28}$, much fewer in Chinese rural areas, which may not completely reflect the characteristics of Chinese rural population. Status of ICH of Chinese rural population needs to be studied independently, considering the racial and ethnic diversity between western and Asia and the economic and geographic differences between rural and urban areas ${ }^{29}$.

Hence, the aim of this study was to explore the prevalence of ideal cardiovascular health according to the definition of AHA and the related factors in Chinese rural population. We performed the current study based on the cohort of the Henan Rural Cohort.

\section{Methods}

\section{Study design and participants}

The study population was selected from the Henan Rural Cohort Study, which is a prospective study of chronic noncommunicable disease with a large sample of rural people established in Henan Province, China during 2015-2017.In brief, the cohort used a multistage stratified cluster sampling method to recruit participantsaged from 18 to 79 years. With a response rate of $93.7 \%, 39259$ individuals were included in the cohort study. Detailed information of the cohort has been described elsewhere ${ }^{30}$. The participants were excluded if they: (1) were diagnosed with CHD $(n=1734)$; (2) were diagnosed with Stroke ( $n=2642)$; (3)were missed information needed in the present study excluded $(n=124)$. Finally, 35018 adults were ultimately included in the study. Before the study commenced, participants were informed of the study's purpose, health benefits, and potential hazards. Participants were required to provide informed consent and both the researchers and respondents agreed to use the data for scientific research purposes only.

\section{Data collection}

A standard questionnaire was conducted by well-trained research staffby face to face interviews to obtain information regarding participants' demographic characteristics, lifestyles, Food Frequency Questionnaire (FFQ),individual history of diseasesand medication. Demographic characteristics included age, gender, educational level (elementary school or below, junior high school and high school or above), marital status (married/cohabitating and unmarried/divorced/widowed), per capita monthly income $(<500,500 \sim$ and $\geq 1000$ renminbi $(\mathrm{RMB}))$. 
Lifestyle factorsincluded smoking (current smoker, non-current smoker), drinking (current drinking, non-current drinker) and physical activity. International Physical Activity Questionnaire (IPAQ 2001) was used to assess the levels of physical activity $^{31}$. The FFQ, covered questions about thirteen main food groups, including staple foods, livestock, poultry, fish, eggs, dairy, fruits, vegetables, beans, nuts, pickles, cereal and animal oil, which based on the Dietary Guidelines for Chinese Residents and the eating habits of Henan people.Participants were asked generally about the frequency (daily, weekly, monthly, yearly, or never) and amount (in liang (50 g)) of their consumption of each food or food group in the past 12 months. Previous studies have shown that the FFQ has good reproducibility and validity ${ }^{32}$. Individual history of disease and medication were self-reported by the participants, including CVD (coronary heart disease, myocardial infarction, stroke, or heart failure), hypertension, diabetes mellitus, hyperlipidemia, and the use of antihypertensive, cholesterollowering, or glucose-lowering medications.

Weight and height were measured twice in light clothing with shoes offand recorded to the nearest $0.1 \mathrm{~kg}$ and $0.1 \mathrm{~cm}$ respectively and we calculated the average of the two measures. Body mass index (BMI) was computed as body weight $(\mathrm{kg})$ divided by height square $\left(\mathrm{m}^{2}\right)$ based on the measurement.WC was also measured twice with a standard tape around the waist about $1 \mathrm{~cm}$ above the navel and parallel to the ground. Blood pressure was measured three times by electronic sphygmomanometer (Omron HEM-7071A, Japan) in the right arm of sitting position after at least 5 min rest. There were 30 s intervals between the three measurements.

The venous blood samples were collected from subjects after overnight fasting for at least 8 hours and stored in $-80^{\circ} \mathrm{C}$ cryogenic refrigerator before analysis. The fasting blood glucose (FBG) was analyzed with via glucose oxidative method (GOD-PAP) by ROCHE Cobas C501 automatic biochemical analyzer ${ }^{30}$. Total cholesterol, triglyceride, high-density lipoprotein cholesterol and low-density lipoprotein cholesterol were measured by Roche Cobas C501 automatic biochemical analyzer.

\section{Definitions}

Tracking disease was easy because all respondents were covered by the New Rural Cooperative Medical System (NRCMS) and each participant had a unique medical insurance card number and ID. NRCMS medical records reviews of CVD were confirmed by rural doctor after self-reporting the history of CVD, and further determined by the outcome committee which is composed of a physician, an endocrinologist, a cardiologist, and an epidemiologist according to standardization recommended by World Health Organization criteria ${ }^{3334}$.

Smoking status was classified into current smoker who smoked more than one cigarette per day in the past six months and non-current smoker including ever smoker and never smoker. Alcohol drinking status was categorized into current drinking who consumed alcoholic drinks for twelve or more times in the past one year, whether spirits, beer, wine or other forms of alcoholic beverage, and non-current drinker including ever drinker and never drinker.WC $<90 \mathrm{~cm}$ for men and WC $<80 \mathrm{~cm}$ for women were classified as normal waist circumference, and WC $\geq 90 \mathrm{~cm}$ for men and WC $\geq 80 \mathrm{~cm}$ for women were classified as abdominal obesity.

\section{Ideal Cardiovascular Health metrics}

According to the AHA definitions of ideal cardiovascular health $(\mathrm{ICH})^{8}$, each of the 7 cardiovascular health metrics (smoking, physical activity, BMI, diet, total cholesterol, blood pressure and fasting plasma glucose) was categorized as ideal and non-ideal, respectively. ICH metrics were as follows: ideal smoking status, never smoker; ideal $\mathrm{BMI}, \mathrm{BMI}<25$ $\mathrm{kg} / \mathrm{m}^{2}$; ideal physical activity (PA), PA $\geq 150 \mathrm{~min} / \mathrm{wk}$ of moderate intensity or $\geq 75 \mathrm{~min} / \mathrm{wk}$ of vigorous intensity or $\geq 150$ $\mathrm{min} /$ wk of moderate-vigorous intensity combination; ideal diet, $\geq 4$ components; ideal total cholesterol (TC), TC $<5.18$ $\mathrm{mmol} / \mathrm{L}$ untreated; ideal blood pressure (BP), BP $<120 /<80 \mathrm{~mm} \mathrm{Hg}$ untreated, and ideal fasting plasma glucose (FPG), $\mathrm{FPG}<5.6 \mathrm{mmol} / \mathrm{L}$ untreated. Furthermore, ICH was defined according to the American Heart Association's 2020 Strategic 
Impact Goals as follows: the simultaneous presence of 4 ideal health behaviors (ideal smoking status, ideal BMI, ideal PA, and ideal diet) and 4 ideal health factors (ideal smoking status, ideal TC, ideal BP, and ideal FPG) in the absence of a history of cardiovascular disease.

We made some adaptations as appropriate for the healthy diet score. Ideal diet was defined as healthy diet score $\geq 4$ components $^{35}$ (fruits and vegetables $\geq 500 \mathrm{~g} / \mathrm{d}$; fish $\geq 200 \mathrm{~g} /$ week; soybean products $\geq 125 \mathrm{~g} / \mathrm{d}$; red meat $<75 \mathrm{~g} / \mathrm{d}$; drinking tea).

\section{Statistical analysis}

All analyses were performed separately for men and women. Continuous variables presented as mean \pm SD were compared by using the t-test or analysis of variance, while categorical variables presented as numbers and proportions were compared by using chi-square test. The prevalence was standardized by using the direct method according to the Chinese Population Census 2010. We calculated the ICH scores by summing the total number of ideal metrics for each participant, ranging from 0 to 7 , and ICH scores was further categorized into Non-CVH (0-4 scores) and Ideal CVH (5-7 scores) based on the total number of ideal indices. Ideal health behaviors (IHB) scores was calculated by summing the total number of IHB metrics ranging from 0 to 4 , and IHB scores was further categorized into Non-HB (0-3 scores) and Ideal HB (4 scores). Ideal health factors (IHF) scores was calculated by summing the total number of IHF metrics ranging from 0 to 4, and IHF scores was further categorized into Non-HF (0-3 scores) and Ideal HF (4 scores) The association between socioeconomic factors and the prevalence of Ideal $\mathrm{CVH}$, Ideal HB and Ideal HF were estimated by the multivariable logistic regression models, and were shown by odds ratio (OR) and $95 \%$ confidence interval (Cl). All selected characteristics were included in multivariable logistic regression models. Besides, the three indices including age groups, educational attainment and per capita monthly income were taken as continuous variables to explore whether there was a linear trend of ORs. A p-value less than 0.05 (two tailed) was applied to assess the statistical significance. Statistical analyses were performed by SPSS software V.26.0 and R version 3.6.3.

\section{Results}

\section{Characteristics of the participants}

The characteristics of the study participants according to sex is presented in Table 1. Among the 35081 participants, $21370(60.92 \%)$ were women. The mean age of participants was 54.72 years with a range from 18 to 79 years. Most of the participants were married $(90.19 \%)$ and only $16.04 \%$ of the participants had high school or above education. The proportion of current smokers was $19.60 \%$, and $50.28 \%$ were current drinkers in men. The mean BMI of participants was $24.79 \pm 3.56 \mathrm{~kg} / \mathrm{m}^{2}$. Compared with men, women were more likely to be of lower educational level, lower proportion of current smokers and current drinkers, lower SBP, DBP and WC, and of higher BMI and TC. The differences in age, education, income, smoking, drinking, SBP, DBP, TC and WC were statically significant between men and women $(P<0.05$ for each).

\section{Prevalence of the ICH metrics}

Fig. 1shows the distribution of components of each ICH metrics. The proportions of ICH metrics of participants were as follows: ideal smoking status, 73.12\% (31.81\% in men and 99.62\% in women); ideal BMI, 54.49\% (56.85\% in men and $52.97 \%$ in women); ideal diet, $0.48 \%$ (0.81\% in men and $0.26 \%$ in women); ideal physical activity, $91.37 \%$ ( $85.85 \%$ in men and $94.91 \%$ in women); ideal total cholesterol, $67.83 \%$ (71.96\% in men and $65.18 \%$ in women); ideal blood pressure, $39.99 \%$ (36.32\% in men and $42.35 \%$ in women); ideal fasting plasma glucose, $71.13 \%$ (70.68\% in men and $71.42 \%$ in women). Obviously, diet (0.48\%) was the lowest prevalence of ICH metrics, whereas physical activity (91.37\%) was the most prevalent, and the features were similar in men. However, the most and least prevalent of ideal metrics in women 
were smoking and diet, respectively. Most study participants reached ideal health status for the following ICH metrics: physical activity, blood glucose, smoking, total cholesterol, and BMI. Apartfromtotal cholesterol, Blood pressure, diet and $\mathrm{BMI}$, each ideal ICH metric in women were significantly higher than men $(P<0.001)$ (Table S1).Fig. 2 presents the prevalence of each ICH metric with age groups. With the increase of age, the proportions of ideal total cholesterol, ideal blood pressure, ideal fasting glucose, ideal smoking status and ideal diet decreased.

\section{The prevalence of the ICH scores, IHB scores and IHF scores}

Only 14 participants $(0.04 \%)$ met 7 scores of $\mathrm{ICH}$, and the prevalence of $\leq 1$ was $2.81 \%$. The age-standardized prevalence of Ideal CVH (ICH scores $\geq 5$ ) was $48.86 \%$ (28.26\% in men and $60.70 \%$ in women). The age-standardized mean levels of the ICH scores was 4.30 (3.62 in men and 4.69 in women). There was significant difference about the ICH scores between men and women $(P<0.001)$. Only $0.12 \%$ of participants had all 4 of IHB scores, and $23.73 \%$ had all 4 of IHF scores. The age-standardized prevalence of 4 of IHB scores and 4 of IHF scores were $0.16 \%(0.17 \%$ in men and $0.16 \%$ in women) and $33.26 \%$ (10.06\% in men and $46.60 \%$ in women), respectively. Furthermore, the percentage of 4 of IHF was higher (19.56\%) than the percentage of 4 of IHB $(0.11 \%)$. The proportions of meeting 4 of IHF scores for women was higher than men $(27.49 \%$ vs. $7.20 \%)$, while the proportions of 4 of IHB scores for women was the same as that of men $(0.11 \%$ vs. $0.11 \%)$ (Table 2).Fig. 3 showsthe distribution of ICH scores, IHB scores and IHF scores by sex. The most prevalence of ICH scores was 4 (4 in men and 4 in women), while the most prevalence of IHB scores and IHF scores were 2 (2 in men and 3 in women) and 3 ( 2 in men and 3 in women), respectively.

The distributions of ICH scores, IHB scores and IHF scores according to age are shown in Fig. 4. The scores of ICH, IHB and IHF were higher in younger age groups. Apart from IHB scores, age had an inverse relationship with the ICH scores and IHF scores in total populations and women. The ICH mean score in women were higher than that in men across all age groups.

\section{Associations of potential risk factors for Ideal CVH, Ideal HF and Ideal HB.}

Table 3 describes the odds ratios (ORs) of potential influencing factors associated with Ideal CVH, Ideal HF and Ideal HB. Much older, men, current drinking and abdominal obesity were significantly positively associated withthe prevalence of Ideal CVH (ICH scores 25 ) and Ideal HF(IHF scores>3). However, age, sex and current drinking were not significantly associated with Ideal HB, and high-level education had a protective effect for Ideal HB. Abdominal obesity was common risk factors for Ideal $\mathrm{CVH}$, Ideal HF and Ideal HB. The risk of abdominal obesity for Ideal CVH was particularly high, and men were much more at risk than women. In addition, women who were much older had a higher risk to Ideal $\mathrm{CVH}$ than men, while current drinking had a lower risk (Table S2). The risk ofcurrent drinkingfor Ideal HBwas not significantly associated for men and women. However, current drinking for Ideal HF increased the risk of men, and reduces the risk of women (Table S3).

\section{Discussion}

The present large survey specialized in Chinese rural population provided important new evidence on the prevalence of ideal cardiovascular health $(\mathrm{ICH})$ in China. Our study shows that the prevalence of $\mathrm{ICH}$ is severe in rural China. Only $0.10 \%$ ( $0.07 \%$ in men and $0.11 \%$ in women) metall $7 \mathrm{ICH}$ metrics, and there was a substantial difference between the percentages of $7 \mathrm{ICH}$ metrics. The ideal physical activity was the highest prevalence of ICH metrics, while the ideal diet was the lowest prevalence of $\mathrm{ICH}$ metrics. only $0.12 \%$ participants met all 4 ideal health behaviors (IHB)metrics, while $23.73 \%$ participants met all 4 Ideal health factors (IHF) metrics. Apart from IHB scores, age had an inverse relationship with the ICH scores and IHF scores in total populations and women, and women had higher prevalence than men in all age ranges.

According to the studies, the proportions of $\mathrm{ICH}$ in the general population in various countries was extremely low. A review including 18 studies in United States showed ICH was very low ranging from 1\% to 12\% with 11 of 15 (73\%) studies 
reporting $\leq 5 \%^{36}$. In a community-based sample from Central Europe, few adults had ideal $\mathrm{CVH}$, and men were more affected by poor $\mathrm{CVH}^{37}$. Results from the National Health Survey showed that only $1 \%$ of Brazilian Population reached $\mathrm{ICH}^{38}$. Only $0.15 \%$ of Australian adults had ideal status on all of the seven metrics among Australian adults ${ }^{39}$. The prevalence of meeting all $7 \mathrm{ICH}$ metrics was $0.67 \%$ among middle-aged Korean men ${ }^{23}$.Similar results were found in our study, and our results complement the research of ICH among Chinese rural population. The prevalence of ICH in $\mathrm{Chinese}$ rural population waslower than Chinese urban population $(0.5 \%)^{26}$ and Chinese general population $(0.2 \%)^{27,28}$.Compared with the percentages of $7 \mathrm{ICH}$ metrics among American adults ${ }^{8}$, the rural Chinese displayed higher ideal percentages of physical activity, BMI, total cholesterol and FPG. Both populations have similar ideal proportions of smoking status, diet and blood pressure, and the proportions of ideal diet was extremely low. In addition, Fasting plasma glucose was the most prevalent ideal metric $(71.2 \%)$, whereas physical activity was the least prevalent $(18.1 \%)$ in Chinese urban population ${ }^{26}$. However, in our findings, the ideal physical activity (91.37\%) was the highest prevalence of ICH metrics, while the ideal diet $(0.48 \%)$ was the lowest prevalence of $\mathrm{ICH}$ metrics in Chinese rural population. In Chinese rural areas, both men and women engage in agricultural activities from sunrise to sunset, with intervening rest, which is associated with higher prevalence of ideal physical activity. It is beneficial to improve the status of TC, FPG, BMI. Rural areas had lower riskfactors burden but higher age-standardized prevalence of CVDs compared with urban areas ${ }^{40,41}$, which might be due to the lower educational level, lower quality of health services, and less frequency of proven therapies used. Previous studies have reported that the prevalence of $\mathrm{ICH}$ was higher in women $26,27,42,43$. Similar results were found in our study.The age-standardized prevalence of ICHin men and women were $0.08 \%$ vs. $0.12 \%$. Thus, men should be taken as a primary risk factor for cardiovascular health in Chinese rural region.

Only $0.17 \%$ and $36.03 \%$ participants met all $4 \mathrm{IHB}$ and all $4 \mathrm{IHF}$, respectively. The higher percentages in IHF than IHB were in agreement with several studies $27,44-47$, while a US study reported a $2.0 \%$ ideal health behaviors' index and a $1.4 \%$ ideal health factors' index ${ }^{48}$, and $0.52 \%$ of Australian adults had all four ideal cardiovascular health factors and $16.38 \%$ had all four ideal cardiovascular health behaviors ${ }^{39}$. Regional differences, socioeconomic level and lifestyle might partly contribute to the differences. The IHB scores, IHF scores and ICH scores were higher in younger age groups,and women had higher prevalence than men in all age ranges. Another interesting phenomenon is that there were different trend changes in the mean of IHB scores, IHF scores and ICH scores according age in men and women. Apart from IHB scores, age had an inverse relationship with the ICH scores and IHF scores in total populations and women. The results of our findings showed that IHB was most likely to achieve the highest yield, especially diet, because they were noted to be least prevalent uniformly across all cohorts studied in many countries. The low percentage of ideal diet was the major reason to the extremely low percentage of $\mathrm{ICH}$. The low prevalence of ideal diet suggests that we should put in more effort to improve the status of diet metrics. Compositive efforts focusing on these targets will accelerate the achievement of ICH goals, which will also indirectly influence attainment of ideal status forBMI, total cholesterol, blood pressure, and fasting glucose metrics. Thus, health behaviors included in the definition of overall cardiovascular health represents a critical step toward implementation of primordial prevention (primary prevention), and it is urgent to promote healthy lifestyle in middle- and older-aged individuals to come up to the ideal cardiovascular health status. There have been a lot of research reports that adherence to healthy lifestyle may substantially lower the burden of cardiovascular diseases ${ }^{49-52}$. Maintaining $\mathrm{ICH}$ from early adulthood results in higher health-related quality of life in middle age ${ }^{10,53}$. Even for patients with diabetes and hypertension, maintaining $\mathrm{ICH}$ is beneficial to reduce the risk of cardiovascular disease ${ }^{51,54}$.

Our study further found that abdominal obesity was associated with a higher risk for Ideal ICH, Ideal HB and Ideal HF. Previous studies have found similar results that abdominal obesity showed higher risks on cardiovascular diseases ${ }^{55,56}$. The rates of abdominal obesity still increased rapidly in the Chinese population, especially in rural population ${ }^{57}$. The agestandardized prevalence of abdominal obesity was $43.71 \%$ in the general Chinese rural adults ${ }^{58}$. Thus, effective measures should be taken to improve the status of prevention and control of abdominal obesity to improve the status of ICH.In addition, we found that drinking may be associated with a more ideal CVH and a more ideal HF in women, but not in men.

Page $7 / 20$ 
In a previous study in U.S, women with light or moderate drinking were more likely to have optimal CVH compared to nondrinking women, which was not seen in men ${ }^{59}$, which was consistent with our study. However, since alcohol and smoking are closely related behaviors, drinkers are more likely to not meet the ideal standard of smoking. There have been some research reports that light to moderate alcohol consumption was associated with increased odds of ideal physical activity and ideal $\mathrm{BMI}^{60-62}$. But the mechanism is not clear and further research is needed.

\section{Strengths And Limitations}

Our study combines the epidemiological characteristics and influencing factors of $\mathrm{ICH}$ according to the relatively large sample size of rural population in China. Standardized investigation tools, training and on-site implementation, as well as adjustments of a wide range of potential confounding factors, ensure the reliability of the analysis. Nevertheless, several limitations should also be considered. First, these findings come from a cross-sectional study, rather than a prospective cohort design, thus do not accurately describe causality. Secondly, some residents, such as college students and migrant workers, were not included in the scope of this study because they were studying or working outside. These people are more likely to be young and healthy and have a higher prevalence of $\mathrm{ICH}$, which may lead to the underestimation of ICH in the rural population. Finally, the result was based on a geographical region of central China, which may not be a representative sample of Chinese rural population. However, the rural population of Henan Province accounts for $9 \%$ of Chinese rural population. Therefore, the results of relatively large rural epidemiological studies could reflect the prevalence of ICH in rural areas of China to some extent.

\section{Conclusion}

The percentage of ideal cardiovascular health is extremely low in rural China. Therefore, there is an increasing need for primordial prevention of unhealthy lifestyles and monitoring high risk factors including older, men, low family income, current drinking and abdominal obesityto promote cardiovascular health in rural China.

\section{Abbreviations}

$\mathrm{ICH}$ : ideal cardiovascular health; $\mathrm{CVH}$ : cardiovascular health; IHB: ideal health behaviors; IHF: ideal health factors; ideal CVH: ICH scores $\geq 5$; ideal HB: IHB scores>3; ideal HF: IHF scores>3; CVD: Cardiovascular disease; AHA: American Heart Association; BMl: body mass index; TC: total cholesterol; BP: blood pressure; FPG: fasting plasma glucose; PA: physical activity.

\section{Declarations}

\section{Acknowledgements}

The authors thank all of the participants, coordinators, and administrators for their support during the research. In addition, the authors would like to thank TankoAbdulai for his critical reading of the manuscript.

\section{Author contributions}

During the research, $\mathrm{XW}$ had full access to all the data in the study. XW analyzed the data and wrote the manuscript. TA corrected the manuscript. CW designed the study. XW, XL, ZL, MN, YH, JH, ZT, ZM, WH, LL, CW conducted the collection of the data. All authors read and approve this version of the article.

\section{Funding}


This research was supported by the Foundation of National Key Program of Research and Development of China (Grant NO: 2016YFC0900803), National Natural Science Foundation of China (Grant NO: 81573243, 81602925), Henan Provincial Science Fund for Distinguished Young Scholars (Grant NO: 164100510021), Science and Technology Innovation Talents Support Plan of Henan Province Colleges and Universities (Grant NO: 14HASTIT035). The funders had no role in the study design, data collection and analysis, decision to publish, or preparation of the manuscript. The funders had no role in the study design, data collection and analysis, decision to publish, or preparation of the manuscript.

\section{Availability of data and material}

The raw data supporting the conclusions of this manuscript will be made available by the authors, without undue reservation, to any qualified researcher.

\section{Ethics approval and consent to participate}

Ethics approval was obtained from the "Zhengzhou University Life Science Ethics Committee", and written informed consent was obtained for all participants. Ethic approval code: [2015] MEC (S128).Written informed consent was obtained from all participants.

\section{Consent for publication}

Not applicable.

\section{Competing Interests}

All authors have read and approved this version of the article, and declared that they had no competing or financial interests to disclosure.

\section{Author details}

${ }^{1}$ Department of Epidemiology and Biostatistics,College of Public Health, Zhengzhou University, Zhengzhou, Henan, $P R$. China. ${ }^{2}$ Department of Global Health, School of Health Sciences, Wuhan University, Wuhan, Hubei, China. ${ }^{3}$ Department of Epidemiology, School of Public Health, Shanxi Medical University, Taiyuan, Shanxi, PR. China.

\section{References}

1. Global, regional, and national disability-adjusted life-years (DALYs) for 359 diseases and injuries and healthy life expectancy (HALE) for 195 countries and territories, 1990-2017: a systematic analysis for the Global Burden of Disease Study 2017. Lancet (London, England) 392, 1859-1922, doi:10.1016/s0140-6736(18)32335-3 (2018).

2. Global, regional, and national age-sex-specific mortality for 282 causes of death in 195 countries and territories, 19802017: a systematic analysis for the Global Burden of Disease Study 2017. Lancet (London, England) 392, 1736-1788, doi:10.1016/s0140-6736(18)32203-7 (2018).

3. Ohira, T. \& Iso, H. Cardiovascular disease epidemiology in Asia: an overview. Circulation journal : official journal of the Japanese Circulation Society 77, 1646-1652, doi:10.1253/circj.cj-13-0702 (2013).

4. Moran, A. et al. Future cardiovascular disease in china: markov model and risk factor scenario projections from the coronary heart disease policy model-china. Circulation. Cardiovascular quality and outcomes 3, 243-252, doi:10.1161/circoutcomes.109.910711 (2010).

5. Nguyen, H. N., Fujiyoshi, A., Abbott, R. D. \& Miura, K. Epidemiology of cardiovascular risk factors in Asian countries. Circulation journal : official journal of the Japanese Circulation Society 77, 2851-2859, doi:10.1253/circj.cj-13-1292 (2013). 
6. Hata, J. \& Kiyohara, Y. Epidemiology of stroke and coronary artery disease in Asia. Circulation journal : official journal of the Japanese Circulation Society 77, 1923-1932, doi:10.1253/circj.cj-13-0786 (2013).

7. Yang, Z. J. et al. Prevalence of cardiovascular disease risk factor in the Chinese population: the 2007-2008 China National Diabetes and Metabolic Disorders Study. European heart journal 33, 213-220, doi:10.1093/eurheartj/ehr205 (2012).

8. Lloyd-Jones, D. M. et al. Defining and setting national goals for cardiovascular health promotion and disease reduction: the American Heart Association's strategic Impact Goal through 2020 and beyond. Circulation 121, 586613, doi:10.1161/CIRCULATIONAHA.109.192703 (2010).

9. Gao, J. et al. Ideal cardiovascular health behaviors and factors prevent the development of hypertension in prehypertensive subjects. Clin Exp Hypertens 37, 650-655, doi:10.3109/10641963.2015.1047938 (2015).

10. Corlin, L., Short, M. I., Vasan, R. S. \& Xanthakis, V. Association of the Duration of Ideal Cardiovascular Health Through Adulthood With Cardiometabolic Outcomes and Mortality in the Framingham Offspring Study. JAMA cardiology, doi:10.1001/jamacardio.2020.0109 (2020).

11. Joseph, J. J. et al. Ideal cardiovascular health, glycaemic status and incident type 2 diabetes mellitus: the REasons for Geographic and Racial Differences in Stroke (REGARDS) study. Diabetologia 62, 426-437, doi:10.1007/s00125018-4792-y (2019).

12. Joseph, J. J. et al. The association of ideal cardiovascular health with incident type 2 diabetes mellitus: the MultiEthnic Study of Atherosclerosis. Diabetologia 59, 1893-1903, doi:10.1007/s00125-016-4003-7 (2016).

13. Ramírez-Vélez, R. et al. Ideal Cardiovascular Health and Incident Cardiovascular Disease Among Adults: A Systematic Review and Meta-analysis. Mayo Clinic proceedings 93, 1589-1599, doi:10.1016/j.mayocp.2018.05.035 (2018).

14. Fang, N., Jiang, M. \& Fan, Y. Ideal cardiovascular health metrics and risk of cardiovascular disease or mortality: A meta-analysis. International journal of cardiology 214, 279-283, doi:10.1016/j.ijcard.2016.03.210 (2016).

15. Lachman, S. et al. Ideal cardiovascular health and risk of cardiovascular events in the EPIC-Norfolk prospective population study. European journal of preventive cardiology 23, 986-994, doi:10.1177/2047487315602015 (2016).

16. Zhou, L. et al. Ideal cardiovascular health metrics and its association with 20-year cardiovascular morbidity and mortality in a Chinese population. Journal of epidemiology and community health $72,752-758$, doi:10.1136/jech2017-210396 (2018).

17. Ommerborn, M. J. et al. Ideal Cardiovascular Health and Incident Cardiovascular Events: The Jackson Heart Study. American journal of preventive medicine 51, 502-506, doi:10.1016/j.amepre.2016.07.003 (2016).

18. Dong, Y. et al. Ideal Cardiovascular Health Status and Risk of Cardiovascular Disease or All-Cause Mortality in Chinese Middle-Aged Population. Angiology 70, 523-529, doi:10.1177/0003319718813448 (2019).

19. Folsom, A. R. et al. Community prevalence of ideal cardiovascular health, by the American Heart Association definition, and relationship with cardiovascular disease incidence. Journal of the American College of Cardiology 57, 1690-1696, doi:10.1016/j.jacc.2010.11.041 (2011).

20. Díez-Espino, J. et al. Impact of Life's Simple 7 on the incidence of major cardiovascular events in high-risk Spanish adults in the PREDIMED study cohort. Revista espanola de cardiologia (English ed.) 73, 205-211, doi:10.1016/j.rec.2019.05.010 (2020).

21. Yang, Q. H. et al. Trends in Cardiovascular Health Metrics and Associations With All-Cause and CVD Mortality Among US Adults. Jama-Journal of the American Medical Association 307, 1273-1283, doi:10.1001/jama.2012.339 (2012).

22. Aneni, E. C. et al. Estimates of Mortality Benefit From Ideal Cardiovascular Health Metrics: A Dose Response MetaAnalysis. Journal of the American Heart Association 6, doi:10.1161/JAHA.117.006904 (2017).

23. Kim, J. Y. et al. Cardiovascular health metrics and all-cause and cardiovascular disease mortality among middle-aged men in Korea: the Seoul male cohort study. Journal of preventive medicine and public health = Yebang Uihakhoe chi 46, 319-328, doi:10.3961/jpmph.2013.46.6.319 (2013). 
24. Rasmussen-Torvik, L. J. et al. Ideal cardiovascular health is inversely associated with incident cancer: the Atherosclerosis Risk In Communities study. Circulation 127, 1270-1275, doi:10.1161/CIRCULATIONAHA.112.001183 (2013).

25. Younus, A. et al. A Systematic Review of the Prevalence and Outcomes of Ideal Cardiovascular Health in US and NonUS Populations. Mayo Clinic proceedings 91, 649-670, doi:10.1016/j.mayocp.2016.01.019 (2016).

26. Zeng, Q. et al. Ideal cardiovascular health in Chinese urban population. International Journal of Cardiology 167, 23112317, doi:10.1016/j.ijcard.2012.06.022 (2013).

27. Bi, Y. et al. Status of Cardiovascular Health in Chinese Adults. Journal of the American College of Cardiology 65, 10131025, doi:10.1016/j.jacc.2014.12.044 (2015).

28. Wu, H.-Y., Sun, Z.-H., Cao, D.-P., Wu, L.-X. \& Zeng, Q. Cardiovascular health status in Chinese adults in urban areas: analysis of the Chinese Health Examination Database 2010. International journal of cardiology 168, 760-764, doi:10.1016/j.ijcard.2012.09.235 (2013).

29. Hartley, D. Rural health disparities, population health, and rural culture. American journal of public health $94,1675-$ 1678, doi:10.2105/ajph.94.10.1675 (2004).

30. Liu, X. et al. The Henan Rural Cohort: a prospective study of chronic non-communicable diseases. International journal of epidemiology, doi:10.1093/ije/dyz039 (2019).

31. Bauman, A. et al. The International Prevalence Study on Physical Activity: results from 20 countries. The international journal of behavioral nutrition and physical activity 6, 21, doi:10.1186/1479-5868-6-21 (2009).

32. Xue, Y. et al. Reproducibility and validity of an FFQ in the Henan Rural Cohort Study. Public health nutrition, 1-7, doi:10.1017/s1368980019002416 (2019).

33. Nomenclature and criteria for diagnosis of ischemic heart disease. Report of the Joint International Society and Federation of Cardiology/World Health Organization task force on standardization of clinical nomenclature. Circulation 59, 607-609, doi:10.1161/01.cir.59.3.607 (1979).

34. Aho, K. et al. Cerebrovascular disease in the community: results of a WHO collaborative study. Bulletin of the World Health Organization 58, 113-130 (1980).

35. Han, C. et al. Ideal cardiovascular health and incidence of atherosclerotic cardiovascular disease among Chinese adults: the China-PAR project. Science China. Life sciences 61, 504-514, doi:10.1007/s11427-018-9281-6 (2018).

36. Younus, A. et al. Prevalence of Ideal Cardiovascular Health Among Adults in the United States. Journal of the American College of Cardiology 66, 1633-1634, doi:10.1016/j.jacc.2015.06.1348 (2015).

37. Medina-Inojosa, J. R. et al. Prevalence of ideal cardiovascular health in a Central European community: results from the Kardiovize Brno 2030 Project. European journal of preventive cardiology 27, 441-443, doi:10.1177/2047487319834875 (2020).

38. Velasquez-Melendez, G. et al. Ideal cardiovascular health prevalence in the Brazilian population - National Health Survey (2013). Revista brasileira de epidemiologia = Brazilian journal of epidemiology 18 Suppl 2, doi:10.1590/19805497201500060009 (2015).

39. Peng, Y. \& Wang, Z. Cardiovascular health status among Australian adults. Clinical epidemiology 10, 167-178, doi:10.2147/CLEP.S155783 (2018).

40. Yan, R., Li, W., Yin, L., Wang, Y. \& Bo, J. Cardiovascular Diseases and Risk-Factor Burden in Urban and Rural Communities in High-, Middle-, and Low-Income Regions of China: A Large Community-Based Epidemiological Study. Journal of the American Heart Association 6, doi:10.1161/JAHA.116.004445 (2017).

41. Wang, Y. et al. Prevalence and Influencing Factors of Coronary Heart Disease and Stroke in Chinese Rural Adults: The Henan Rural Cohort Study. Frontiers in public health 7, 411, doi:10.3389/fpubh.2019.00411 (2019). 
42. Simon, M. et al. Sex disparities in ideal cardiovascular health. Heart (British Cardiac Society) 103, 1595-1601, doi:10.1136/heartjnl-2017-311311 (2017).

43. Magodoro, I. M. et al. Female sex and cardiovascular disease risk in rural Uganda: a cross-sectional, population-based study. BMC cardiovascular disorders 19, 96, doi:10.1186/s12872-019-1072-9 (2019).

44. Graciani, A., León-Muñoz, L. M., Guallar-Castillón, P., Rodríguez-Artalejo, F. \& Banegas, J. R. Cardiovascular health in a southern Mediterranean European country: a nationwide population-based study. Circulation. Cardiovascular quality and outcomes 6, 90-98, doi:10.1161/CIRCOUTCOMES.112.967893 (2013).

45. Zhao, Y. et al. Status of cardiovascular health among adults in a rural area of Northwest China: Results from a crosssectional study. Medicine 95, e4245, doi:10.1097/MD.0000000000004245 (2016).

46. Janković, S., Stojisavljević, D., Janković, J., Erić, M. \& Marinković, J. Status of cardiovascular health in a transition European country: findings from a population-based cross-sectional study. International journal of public health 59 , 769-778, doi:10.1007/s00038-014-0579-1 (2014).

47. Moghaddam, M. M. et al. Distribution of ideal cardiovascular health in a community-based cohort of Middle East population. Annals of Saudi medicine 34, 134-142, doi:10.5144/0256-4947.2014.134 (2014).

48. Bambs, C. et al. Low prevalence of "ideal cardiovascular health" in a community-based population: the heart strategies concentrating on risk evaluation (Heart SCORE) study. Circulation 123, 850-857, doi:10.1161/CIRCULATIONAHA.110.980151 (2011).

49. Lv, J. et al. Adherence to Healthy Lifestyle and Cardiovascular Diseases in the Chinese Population. Journal of the American College of Cardiology 69, 1116-1125, doi:10.1016/j.jacc.2016.11.076 (2017).

50. Larsson, S. C., Akesson, A. \& Wolk, A. Healthy diet and lifestyle and risk of stroke in a prospective cohort of women. Neurology 83, 1699-1704, doi:10.1212/WNL.0000000000000954 (2014).

51. Liu, G. et al. Influence of Lifestyle on IncidentCardiovascular Disease and Mortality in Patients With DiabetesMellitus. Journal of the American College of Cardiology 71, 2867-2876, doi:10.1016/j.jacc.2018.04.027 (2018).

52. Barbaresko, J., Rienks, J. \& Nöthlings, U. Lifestyle Indices and Cardiovascular Disease Risk: A Meta-analysis. American journal of preventive medicine 55, 555-564, doi:10.1016/j.amepre.2018.04.046 (2018).

53. Pool, L. R. et al. Association of cardiovascular health through early adulthood and health-related quality of life in middle age: The Coronary Artery Risk Development in Young Adults (CARDIA) Study. Preventive medicine 126, 105772, doi:10.1016/j.ypmed.2019.105772 (2019).

54. Xue, H. et al. Association of ideal cardiovascular metrics and serum high-sensitivity C-reactive protein in hypertensive population. PloS one 8, e81597, doi:10.1371/journal.pone.0081597 (2013).

55. Goh, V. H. H. \& Hart, W. G. Excess fat in the abdomen but not general obesity is associated with poorer metabolic and cardiovascular health in premenopausal and postmenopausal Asian women. Maturitas 107, 33-38, doi:10.1016/j.maturitas.2017.10.002 (2018).

56. Wildman, R. P. et al. Cardiovascular disease risk of abdominal obesity vs. metabolic abnormalities. Obesity (Silver Spring) 19, 853-860, doi:10.1038/oby.2010.168 (2011).

57. Mi, Y.-J. et al. Prevalence and Secular Trends in Obesity Among Chinese Adults, 1991-2011. American journal of preventive medicine 49, 661-669, doi:10.1016/j.amepre.2015.05.005 (2015).

58. Liu, X. et al. Prevalence and influencing factors of overweight and obesity in a Chinese rural population: the Henan Rural Cohort Study. Scientific reports 8, 13101, doi:10.1038/s41598-018-31336-2 (2018).

59. Ogunmoroti, O. et al. Alcohol and ideal cardiovascular health: The Multi-Ethnic Study of Atherosclerosis. Clinical cardiology 42, 151-158, doi:10.1002/clc.23125 (2019).

60. Dodge, T., Clarke, P. \& Dwan, R. The Relationship Between Physical Activity and Alcohol Use Among Adults in the United States. Am J Health Promot 31, doi:10.1177/0890117116664710 (2017).

Page $12 / 20$ 
61. Traversy, G. \& Chaput, J.-P. Alcohol Consumption and Obesity: An Update. Current obesity reports 4, 122-130, doi:10.1007/s13679-014-0129-4 (2015).

62. Xu, X. et al. [Study on correlation between alcohol consumption and obesity in adults in China]. Zhonghua liu xing bing xue za zhi = Zhonghua liuxingbingxue zazhi 40, 759-764, doi:10.3760/cma.j.issn.0254-6450.2019.07.005 (2019).

\section{Tables}

Table 1. Characteristics of the participants. 


\begin{tabular}{|c|c|c|c|c|}
\hline Variables & Total $(n=35018)$ & $\begin{array}{l}\text { Men(n = } \\
13711)\end{array}$ & $\begin{array}{l}\text { Women }(n= \\
21370)\end{array}$ & $P$ \\
\hline Age (years, mean \pm SD) & $54.74 \pm 12.28$ & $55.74 \pm 12.43$ & $54.10 \pm 12.14$ & $<0.001$ \\
\hline Marital status, $n(\%)$ & & & & 0.706 \\
\hline Married/cohabiting & 31639(90.19) & $12376(90.26)$ & 19263(90.14) & \\
\hline Unmarried/divorced/widowed & $3442(9.81)$ & 1335(9.74) & $2107(9.86)$ & \\
\hline Education level, n (\%) & & & & $<0.001$ \\
\hline Primary school or illiteracy & $15143(43.17)$ & $4460(32.53)$ & 10683(49.99) & \\
\hline Junior high school & $14311(40.79)$ & $6416(46.79)$ & 7895(39.94) & \\
\hline High school or above & $5627(16.04)$ & 2835(20.68) & 2792(13.07) & \\
\hline $\begin{array}{l}\text { Income (RMB per month), n } \\
(\%)\end{array}$ & & & & $<0.001$ \\
\hline$<500$ & $12180(34.72)$ & $4832(35.24)$ & 7348(34.38) & \\
\hline 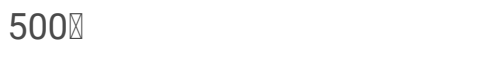 & $11630(33.15)$ & 4376(31.92) & 72.54(33.94) & \\
\hline 1000区 & 11271(32.13) & $4503(32.84)$ & 6768(31.67) & \\
\hline Smoking, n (\%) & & & & $<0.001$ \\
\hline No smoking & 28205(80.40) & $6894(50.28)$ & 21311(99.72) & \\
\hline Current smoking & $6876(19.60)$ & $6817(49.72)$ & $56(0.28)$ & \\
\hline Drinking, n (\%) & & & & $<0.001$ \\
\hline No drinking & 27101(77.25) & $6367(46.44)$ & 20734(97.02) & \\
\hline Current drinking & $7980(22.75)$ & 7344(53.56) & 636(2.98) & \\
\hline $\mathrm{BMI}(\mathrm{kg} / \mathrm{m} 2$ mean $\pm \mathrm{SD})$ & $24.79 \pm 3.56$ & $24.51 \pm 3.49$ & $24.97 \pm 3.59$ & 0.312 \\
\hline $\mathrm{SBP}(\mathrm{mmHg}$, mean $\pm \mathrm{SD})$ & $\begin{array}{l}125.09 \pm \\
19.72\end{array}$ & $125.92 \pm 18.26$ & $124.56 \pm 20.59$ & $<0.001$ \\
\hline $\mathrm{DBP}(\mathrm{mmHg}$, mean $\pm \mathrm{SD})$ & $77.47 \pm 11.60$ & $78.64 \pm 11.94$ & $76.72 \pm 11.32$ & $<0.001$ \\
\hline $\mathrm{TC}(\mathrm{mmol} / \mathrm{L}$, mean $\pm \mathrm{SD})$ & $4.76 \pm 0.98$ & $4.66 \pm 0.95$ & $4.82 \pm 0.99$ & $<0.001$ \\
\hline FPG $(\mathrm{mmol} / \mathrm{L}$, mean $\pm \mathrm{SD})$ & $5.52 \pm 1.49$ & $5.51 \pm 1.49$ & $5.52 \pm 1.49$ & 0.157 \\
\hline WC (cm, mean \pm SD) & $83.90 \pm 10.38$ & $85.46 \pm 10.59$ & $82.90 \pm 10.12$ & $<0.001$ \\
\hline
\end{tabular}

SD, standard deviation; BMI, body mass index; SBP, systolic blood pressure; DBP, diastolic blood pressure; TC, total cholesterol; FPG, fasting plasma glucose. Income (RMB per month), Per capita monthly income. Values are means and standard deviation for continuous variables, and numbers and percentages for categorical variables.

Table 2. Prevalence of the ICH scores, IHB scores and IHF scores by sex. 


\begin{tabular}{|c|c|c|c|c|}
\hline Ideal scores & $\begin{array}{l}\text { Total }(n= \\
27745)\end{array}$ & $\begin{array}{l}\operatorname{Men}(n= \\
11013)\end{array}$ & $\begin{array}{l}\text { Women }(n= \\
16732)\end{array}$ & $P$ \\
\hline IHB scores ${ }^{1}, \mathrm{n}(\%)$ & & & & $<0.001$ \\
\hline 0 & $601(1.71)$ & $598(4.36)$ & $3(0.01)$ & \\
\hline 1 & $4750(13.54)$ & $4250(31.00)$ & $500(2.34)$ & \\
\hline 2 & $16993(48.44)$ & $6815(49.70)$ & $10178(47.63)$ & \\
\hline 3 & $12699(36.20)$ & $2033(14.83)$ & 10666(49.91) & \\
\hline 4 & $38(0.11)$ & $15(0.11)$ & $23(0.11)$ & \\
\hline IHF scores ${ }^{2}, \mathrm{n}(\%)$ & & & & $<0.001$ \\
\hline 0 & $890(2.54)$ & $872(6.36)$ & $18(0.08)$ & \\
\hline 1 & $5186(14.78)$ & $2818(20.55)$ & $2368(11.08)$ & \\
\hline 2 & $10634(30.31)$ & $4969(36.24)$ & $5665(26.51)$ & \\
\hline 3 & $11509(32.81)$ & $4065(29.65)$ & 7444(34.83) & \\
\hline 4 & $6862(19.56)$ & $987(7.20)$ & $5875(27.49)$ & \\
\hline ICH scores ${ }^{3}, \mathrm{n}(\%)$ & & & & $<0.001$ \\
\hline 0 & $106(0.30)$ & $105(0.77)$ & $1(0.003)$ & \\
\hline 1 & $881(2.51)$ & $761(5.55)$ & $120(0.56)$ & \\
\hline 2 & $3689(10.52)$ & 2073(15.12) & $1616(7.56)$ & \\
\hline 3 & 7643(21.79) & $3512(25.61)$ & $4131(19.33)$ & \\
\hline 4 & $9749(27.79)$ & $3851(28.09)$ & $5859(27.60)$ & \\
\hline 5 & $8513(24.27)$ & $2730(19.91)$ & $5783(27.06)$ & \\
\hline 6 & $4486(12.79)$ & $674(4.92)$ & 3812(17.84) & \\
\hline 7 & $14(0.04)$ & $5(0.04)$ & $9(0.04)$ & \\
\hline ICH group, n (\%) & & & & $<0.001$ \\
\hline Ideal $\mathrm{CVH}^{4}$ & 13013(37.09) & $3409(24.86)$ & $9604(44.94)$ & \\
\hline IntermediateCVH ${ }^{5}$ & $17392(49.58)$ & $7363(53.70)$ & $10029(46.93)$ & \\
\hline Poor $\mathrm{CVH}^{6}$ & $4676(13.33)$ & $2939(21.44)$ & $1737(8.13)$ & \\
\hline $\begin{array}{l}\text { ICH scores (mean } \pm \\
\text { SD) }\end{array}$ & $3.98 \pm 1.29$ & $3.54 \pm 1.29$ & $4.27 \pm 1.21$ & $<0.001$ \\
\hline
\end{tabular}

SD, standard deviation; ${ }^{1}$, Ideal health behaviors(IHB) scores; ${ }^{2}$, Ideal health factors(IHF) scores, ${ }^{3}$, ideal cardiovascular health(ICH) scores; ${ }^{4}, 5-7$ scores of $\mathrm{ICH}^{5}{ }^{5}, 3-4$ scores of $\mathrm{ICH}^{6}{ }^{6}, 0-2$ scores of $\mathrm{ICH}$. 
Table 3. The odds ratio and 95\% confidence intervals of Ideal CVH, Ideal HB and Ideal HF.

\begin{tabular}{|c|c|c|c|}
\hline \multirow[t]{2}{*}{ Variables } & Ideal $\mathrm{CVH}^{1}$ & Ideal HB ${ }^{2}$ & Ideal HF ${ }^{3}$ \\
\hline & OR $(95 \% \mathrm{Cl})$ & OR $(95 \% \mathrm{Cl})$ & OR $(95 \% \mathrm{Cl})$ \\
\hline \multicolumn{4}{|l|}{ Age } \\
\hline $18 \rrbracket$ & 1.00 & 1.00 & 1.00 \\
\hline $30 \rrbracket$ & $1.28(1.10-1.50)$ & $1.57(0.33-7.45)$ & $1.65(1.41-1.91)$ \\
\hline $40 \rrbracket$ & $1.93(1.67-2.23)$ & $0.63(0.18-2.22)$ & $2.88(2.50-3.33)$ \\
\hline $50 \rrbracket$ & $3.19(2.77-3.67)$ & $1.45(0.39-5.37)$ & $6.41(5.56-7.39)$ \\
\hline $60 \rrbracket$ & $4.47(3.86-5.17)$ & $1.27(0.32-5.02)$ & $11.59(9.95-13.50)$ \\
\hline $70 \bigotimes 79$ & $5.31(4.52-6.24)$ & $2.09(0.33-13.34)$ & 16.54(13.71-19.96) \\
\hline$P_{\text {trend }}$ & $<0.001$ & 0.334 & $<0.001$ \\
\hline \multicolumn{4}{|l|}{ Sex } \\
\hline men & $3.86(3.61-4.14)$ & $1.26(0.56-2.86)$ & $4.72(4.31-5.17)$ \\
\hline \multicolumn{4}{|l|}{ Marital status } \\
\hline Married/cohabiting & 1.00 & 1.00 & 1.00 \\
\hline Unmarried/divorced/widowed & $1.01(0.92-1.10)$ & $0.48(0.19-1.26)$ & $1.04(0.92-1.16)$ \\
\hline \multicolumn{4}{|l|}{ Income (RMB per month) } \\
\hline$<500$ & 1.00 & 1.00 & 1.00 \\
\hline $500 \rrbracket$ & 1.07(1.01-1.34) & $1.79(0.74-4.37)$ & $1.09(1.01-1.17)$ \\
\hline 10008 & $1.00(0.94-1.07)$ & $0.96(0.45-2.04)$ & $1.06(0.98-1.14)$ \\
\hline$P$ trend & 0.006 & 0.026 & 0.027 \\
\hline \multicolumn{4}{|l|}{ Education level } \\
\hline Primary school or illiteracy & 1.00 & 1.00 & 1.00 \\
\hline Junior high school & $0.95(0.90-1.01)$ & $0.56(0.23-1.36)$ & $0.97(0.90-1.04)$ \\
\hline High school or above & $1.15(1.06-1.25)$ & $0.28(0.11-0.78)$ & $1.11(1.01-1.23)$ \\
\hline$P$ trend & 0.693 & 0.812 & 0.095 \\
\hline \multicolumn{4}{|l|}{ Drinking } \\
\hline No drinking & 1.00 & 1.00 & 1.00 \\
\hline Current drinking & $1.58(1.47-1.72)$ & $1.22(0.47-3.16)$ & $2.05(1.82-2.30)$ \\
\hline Abdominal obesity & $6.79(6.42-7.18)$ & $3.26(1.51-7.02)$ & $2.59(2.44-2.75)$ \\
\hline
\end{tabular}

1, ICH scores $\geq 5 ;{ }^{2}$, IHB scores $>3 ;{ }^{3}$, IHF scores $>3$. OR: odds ratios, $\mathrm{Cl}$ : confidence intervals. Abdominal obesity was defined according to guidelines of the International Diabetes Federation for Chinese populations as a WC $\geq 90 \mathrm{~cm}$ for men and $\geq 80 \mathrm{~cm}$ for women. Normal WC was defined as a WC $<90 \mathrm{~cm}$ for men and $<80 \mathrm{~cm}$ for women ${ }^{48}$. 


\section{Figures}

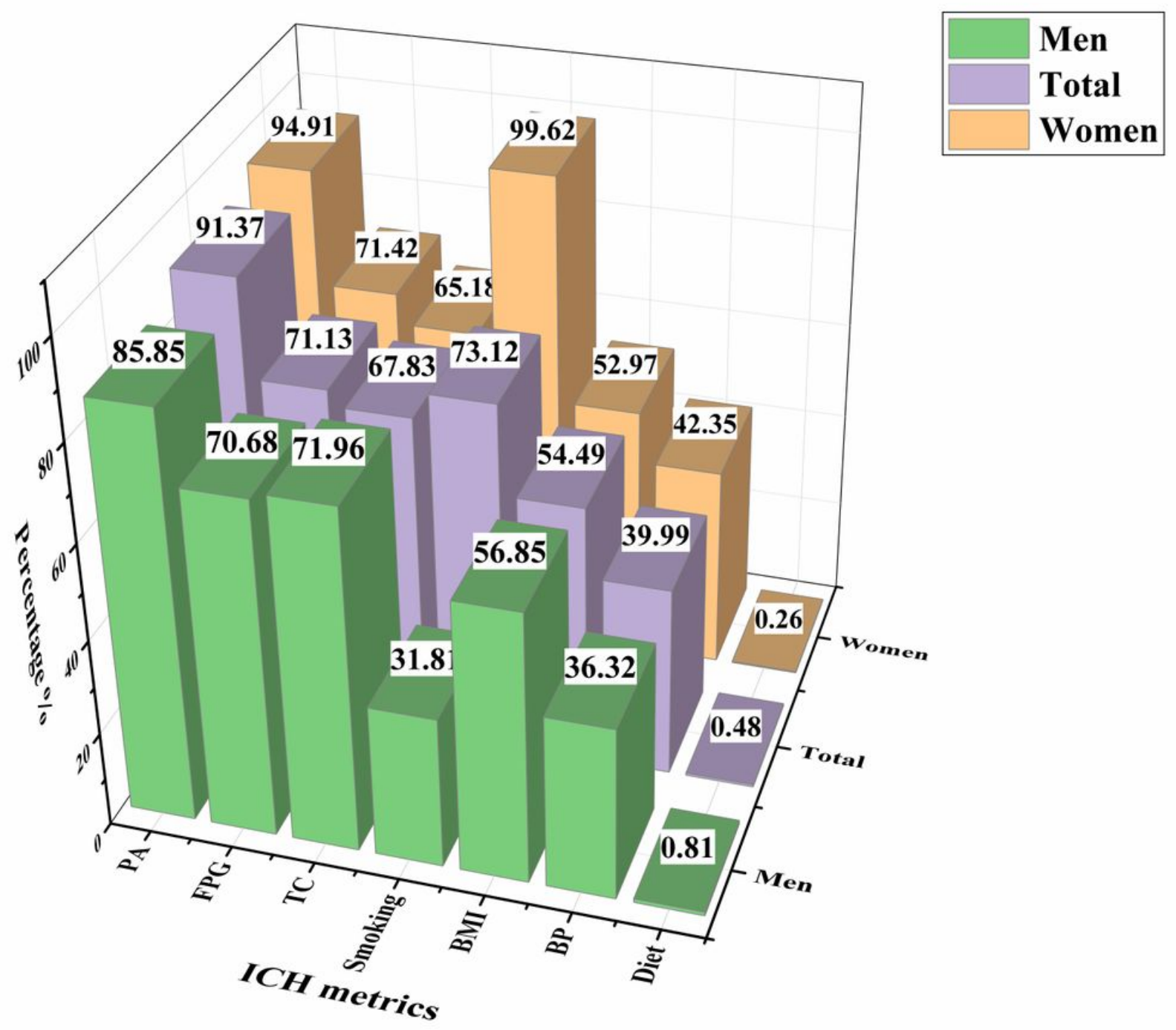

Figure 1

Prevalence of ideal cardiovascular health Metrics $(n=27,745)$. PA, physical activity; BP, blood pressure; FPG, fasting plasma glucose; BMI, body mass index. ICH, Ideal cardiovascular health. 


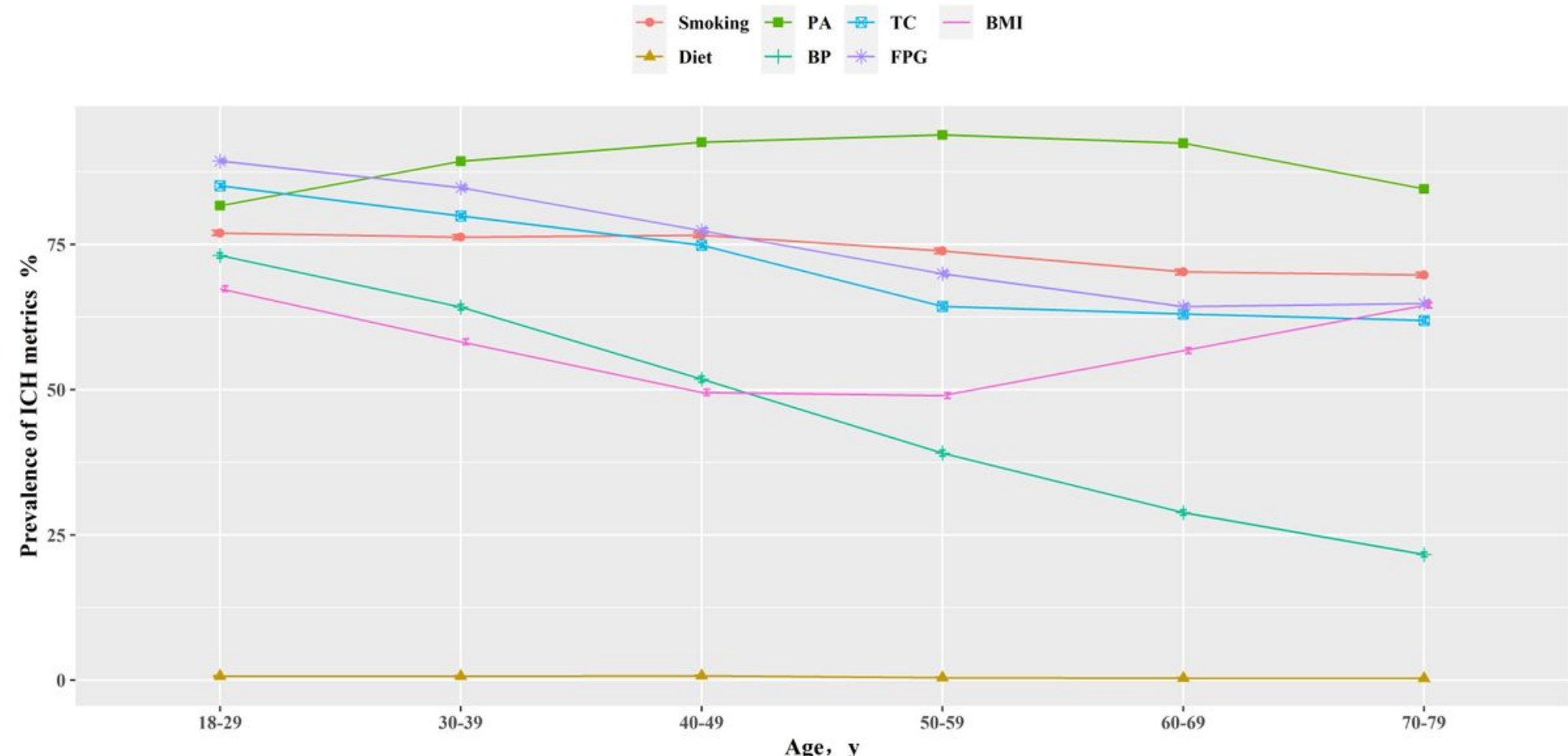

Figure 2

The age-standardized prevalence of ideal cardiovascular health Metrics according to age groups. PA, physical activity; BP, blood pressure; FPG, fasting plasma glucose; BMI, body mass index. 

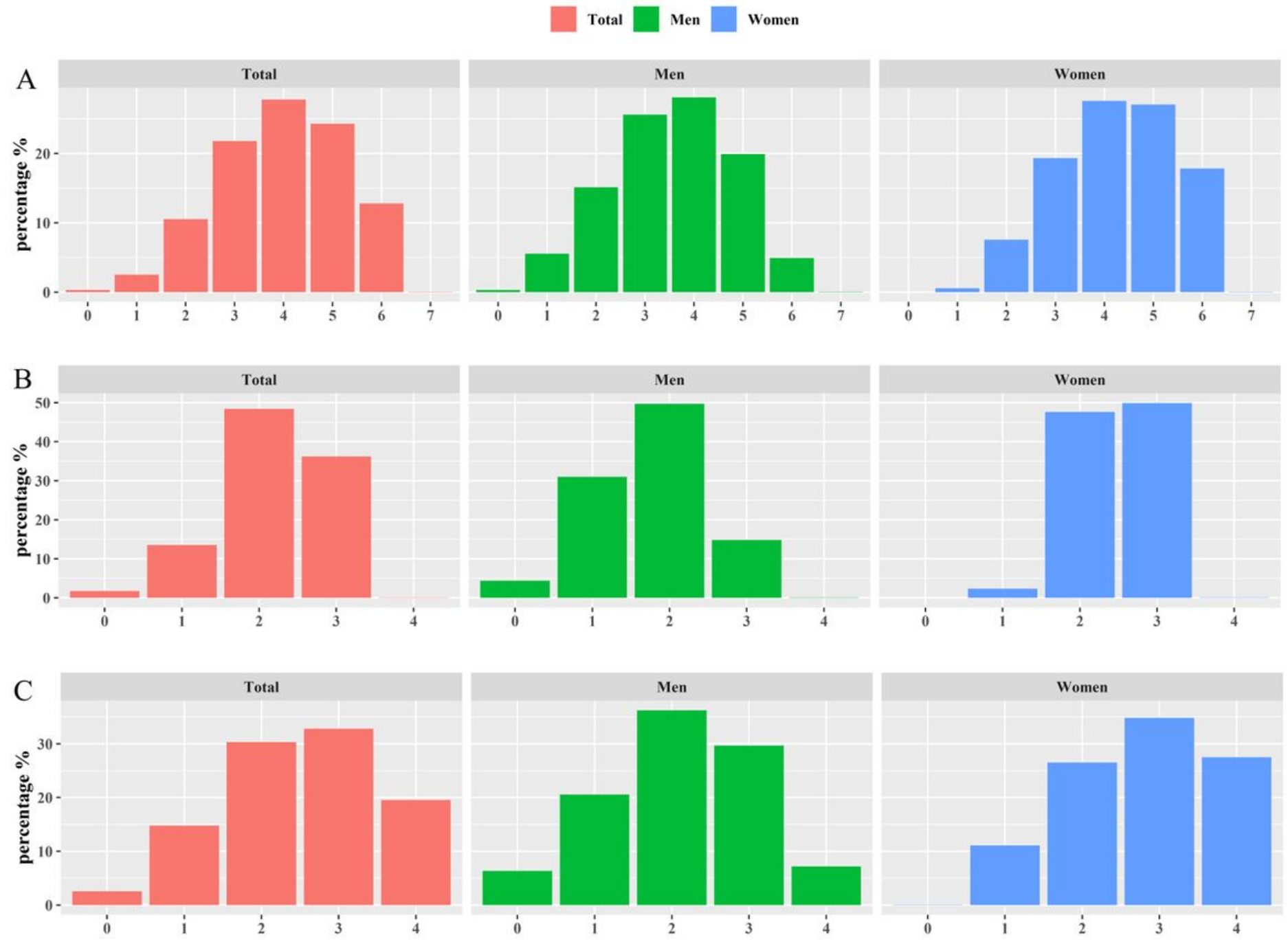

\section{Figure 3}

The distribution of ICH scores, IHB scores and IHF scores by sex. A, ICH scores, ideal cardiovascular health scores; B, IHB scores, ideal health behaviors scores; C, IHF scores, ideal health factors scores. 


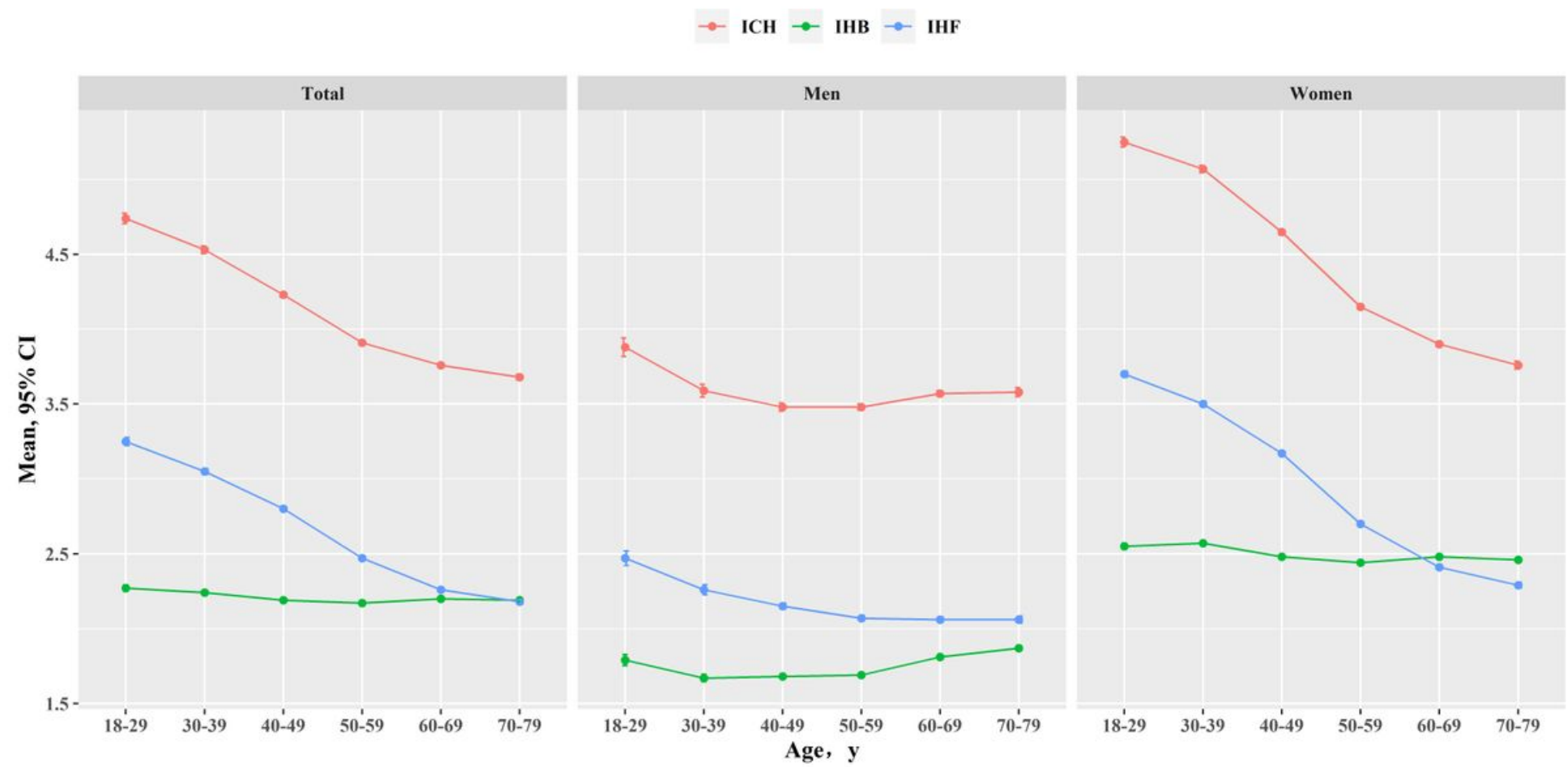

Figure 4

The mean $(95 \% \mathrm{Cl})$ of IHB scores, IHF scores and ICH scores between different age groups for men and women. Error bars indicate $95 \%$ confidence intervals. IHB, ideal health behaviors scores; IHF, ideal health factors scores; ICH, ideal cardiovascular health.

\section{Supplementary Files}

This is a list of supplementary files associated with this preprint. Click to download.

- SupplementaryTables.docx

- Checklistcrosssectional.doc 